\title{
GROWTH THEOREMS FOR HOMOGENEOUS SECOND-ORDER DIFFERENCE EQUATIONS
}

\author{
STEVO STEVIĆ
}

(Received 15 October 1999; revised 19 June 2000)

\begin{abstract}
In this paper we investigate the boundedness and asymptotic behaviour of the solutions of a class of homogeneous second-order difference equations with a single non-constant coefficient. These equations model, for example, the amplitude of oscillation of the weights on a discretely weighted vibrating string. We present several growth theorems. Two examples are also given.
\end{abstract}

\section{Introduction}

In this paper we shall study the second-order linear difference equation of the form

$$
x_{n+1}+b_{n} x_{n}+x_{n-1}=0, \quad n \in \mathbf{N},
$$

where $x_{n}$ is the desired solution and $b_{n}$ is a given real sequence. We shall investigate the boundedness and asymptotic behaviour of the solution of (1).

This equation models, for example, the amplitude of oscillation of the weights on a discretely weighted vibrating string [1, pp. 15-17].

Results for similar problems for second-order differential equations can be found in [3].

If $b_{n}=-2, n \in \mathrm{N}$, we have $x_{n+1}-2 x_{n}+x_{n-1}=0$. This equation has a general solution in the form $a n+b$, where $a, b$ are arbitrary real numbers and thus has unbounded solutions. It is well-known that if $b_{n}=d \in \mathbf{R}, n \in \mathbf{N}$, where $d \geq 2$ or $d \leq-2$, then the equation also has unbounded solutions. This motivates us to investigate the cases when $-2<b_{n}<2, n \in \mathbf{N}$, especially when $b_{n} \rightarrow 0$ as $n \rightarrow \infty$.

\footnotetext{
${ }^{1}$ Matematički Fakultet, Studentski Trg 16, 11000 Beograd, Yugoslavia; e-mail: sstevo@matf.bg.ac.yu. (C) Australian Mathematical Society 2002, Serial-fee code 1446-1811/02
} 
The following "symmetry principle" is very useful in the consideration of (1). If we make the change $y_{n}=(-1)^{n} x_{n}$, (1) becomes

$$
(-1)^{n+1}\left(y_{n+1}-b_{n} y_{n}+y_{n-1}\right)=0, \quad n \in \mathbf{N},
$$

that is,

$$
y_{n+1}-b_{n} y_{n}+y_{n-1}=0, \quad n \in \mathbf{N} \text {. }
$$

Thus it is enough to investigate the cases when $-2<b_{n}<0, n \in \mathbf{N}$. For example, if we show that, under some conditions, when $-2<b_{n}<0, n \in \mathrm{N}$, (1) has either bounded or unbounded solutions, then this also holds for (1) when $0<b_{n}<2, n \in \mathbf{N}$.

Using the substitution $b_{n}=-2 /\left(1+c_{n}\right)$ we may transform the relation of interest into

$$
x_{n+1}-2 x_{n}+x_{n-1}+c_{n}\left(x_{n+1}+x_{n-1}\right)=0, \quad n \in \mathbf{N},
$$

which is in a more suitable form for the calculations which follow (see also [8]).

\section{Preliminaries}

For investigation of the boundedness and asymptotic behaviour of the solution $x_{n}$, we will need a few auxiliary lemmas. The first of these is a discrete variant of the Bellman-Gronwall lemma. The continuous case of this lemma can be found in [2] and [6].

LEMMA 1. If $x_{n}, c_{n} \geq 0, c$ is a positive constant and $x_{n} \leq c+\sum_{i=1}^{n-1} c_{i} x_{i}, n \in \mathbf{N}$, then $x_{n} \leq c \exp \left(\sum_{i=1}^{n-1} c_{i}\right), n \in \mathbf{N}$.

Proof of this lemma and further generalizations can be found in [4] (see also [8]).

LEMMA 2. Let $c_{n}, n \in \mathbf{N} \cup\{0\}$, be a positive sequence and $x_{n}$ be a solution of the difference equation (2). Then

$$
\begin{aligned}
& \left(x_{n+1}-x_{n}\right)^{2}+c_{n} x_{n+1}^{2}+c_{n-1} x_{n}^{2} \\
& \quad=\left(x_{1}-x_{0}\right)^{2}+c_{1} x_{0}^{2}+c_{0} x_{1}^{2}+\sum_{i=1}^{n-1}\left(c_{i+1}-c_{i-1}\right) x_{i}^{2}, \quad n \in \mathbf{N} .
\end{aligned}
$$

Proof. Multiplying (2) by $x_{n+1}-x_{n-1}=x_{n+1}-x_{n}+x_{n}-x_{n-1}$ we get

$$
\left(x_{n+1}-x_{n}\right)^{2}-\left(x_{n}-x_{n-1}\right)^{2}+c_{n}\left(x_{n+1}^{2}-x_{n-1}^{2}\right)=0, \quad n \in \mathbf{N} \text {. }
$$


It follows from (4) that

$$
\sum_{i=1}^{n}\left[\left(x_{i+1}-x_{i}\right)^{2}-\left(x_{i}-x_{i-1}\right)^{2}\right]+\sum_{i=1}^{n} c_{i}\left(x_{i+1}^{2}-x_{i-1}^{2}\right)=0 .
$$

Hence for all $n \in \mathbf{N}$ we obtain (3).

\section{Main results}

We are now in a position to formulate and to prove the main results of this paper. From hereafter we shall exclude the trivial solution from our considerations.

THEOREM 1. Let $c_{n}, n \in \mathbf{N} \cup\{0\}$, be a positive nonincreasing sequence and $x_{n}$ be a solution of (2). Then the sequences $x_{n+1}-x_{n}$ and $c_{n-1} x_{n}^{2}$ are bounded. Further, if $\lim _{n \rightarrow \infty} c_{n}>0$, then $x_{n}$ is bounded.

PROof. From (3) we have

$$
\left(x_{n+1}-x_{n}\right)^{2}+c_{n} x_{n+1}^{2}+c_{n-1} x_{n}^{2} \leq\left(x_{1}-x_{0}\right)^{2}+c_{1} x_{0}^{2}+c_{0} x_{1}^{2}
$$

since $\sum_{i=1}^{n-1}\left(c_{i+1}-c_{i-1}\right) x_{i}^{2} \leq 0$. From that we have the first part of our theorem. In particular, $c_{n-1} x_{n}^{2} \leq\left(x_{1}-x_{0}\right)^{2}+c_{1} x_{0}^{2}+c_{0} x_{1}^{2}=M$, for all $n \in \mathbf{N}$. Therefore if $\lim _{n \rightarrow \infty} c_{n}>0$, we have

$$
x_{n}^{2} \leq \frac{M}{c_{n-1}} \leq \frac{M}{\lim _{n \rightarrow \infty} c_{n}}<+\infty .
$$

Thus the second part of our theorem follows.

THEOREM 2. Let $c_{n}, n \in \mathbf{N} \cup\{0\}$, be a positive nondecreasing sequence such that $c_{n} \geq \delta$ for $n \geq n_{0}$ and $x_{n}$ be a solution of (2). Then $\lim \sup _{n \rightarrow \infty} c_{n} x_{n}^{2}>0$.

PROOF. Without loss of generality we may suppose that $n_{0}=1$. From (3) we get

$$
\left(x_{n+1}-x_{n}\right)^{2}+c_{n} x_{n+1}^{2}+c_{n-1} x_{n}^{2} \geq\left(x_{1}-x_{0}\right)^{2}+c_{1} x_{0}^{2}+c_{0} x_{1}^{2} .
$$

On the other hand $\left(x_{1}-x_{0}\right)^{2}+c_{1} x_{0}^{2}+c_{0} x_{1}^{2}>0$, since we may suppose that $x_{0}$ and $x_{1}$ are not both equal to zero at the same time.

By the inequality between the arithmetic and geometric means and since $c_{n} \geq \delta$, we have

$$
\left(x_{n+1}-x_{n}\right)^{2} \leq 2\left(x_{n+1}^{2}+x_{n}^{2}\right) \leq \frac{2}{\delta}\left(c_{n} x_{n+1}^{2}+c_{n-1} x_{n}^{2}\right), \quad n \in \mathbf{N} .
$$


From all of the above we obtain

$$
\left(1+\frac{2}{\delta}\right)\left(c_{n} x_{n+1}^{2}+c_{n-1} x_{n}^{2}\right) \geq\left(x_{1}-x_{0}\right)^{2}+c_{1} x_{0}^{2}+c_{0} x_{1}^{2}>0, \quad n \in \mathbf{N}
$$

Letting $n \rightarrow \infty$ in the last inequality we obtain that

$$
\left(2+\frac{4}{\delta}\right) \limsup _{n \rightarrow \infty} c_{n} x_{n}^{2} \geq\left(x_{1}-x_{0}\right)^{2}+c_{1} x_{0}^{2}+c_{0} x_{1}^{2}>0
$$

Thus the theorem follows.

The following theorem was essentially proved in [8]. In order to make this paper more complete we shall present its proof here.

THEOREM 3. Let $c_{n}, n \in \mathbf{N} \cup\{0\}$, be a sequence such that $c_{n} \geq \delta>0, n \in \mathbf{N}$ and $\sum_{i=1}^{+\infty}\left|c_{i+1}-c_{i-1}\right|<\infty$. Then all the solutions of (2) are bounded.

Proof. From (3) we have

$$
c_{n-1} x_{n}^{2} \leq\left(x_{1}-x_{0}\right)^{2}+c_{1} x_{0}^{2}+c_{0} x_{1}^{2}+\sum_{i=1}^{n-1}\left|c_{i+1}-c_{i-1}\right| x_{i}^{2}, \quad n \in \mathbf{N}
$$

since $c_{i}>0$.

Since $c_{n} \geq \delta>0, n \in \mathbf{N}$, by the Bellman-Gronwall lemma we get

$$
x_{n}^{2} \leq \frac{\left(x_{1}-x_{0}\right)^{2}+c_{1} x_{0}^{2}+c_{0} x_{1}^{2}}{\delta} \exp \left(\frac{1}{\delta} \sum_{i=1}^{n-1}\left|c_{i+1}-c_{i-1}\right|\right) .
$$

Hence

$$
x_{n}^{2} \leq \frac{\left(x_{1}-x_{0}\right)^{2}+c_{1} x_{0}^{2}+c_{0} x_{1}^{2}}{\delta} \exp \left(\frac{1}{\delta} \sum_{i=1}^{+\infty}\left|c_{i+1}-c_{i-1}\right|\right)<+\infty .
$$

Therefore all solutions of (2) are bounded.

THEOREM 4. Let $c_{n}, n \in \mathbf{N} \cup\{0\}$, be a positive nondecreasing sequence such that $1 \leq m \leq c_{n+1} / c_{n} \leq M<\infty, n \in \mathbf{N} \cup\{0\}$ and $x_{n}$ be a solution of (2). Then

$$
x_{n}^{2}=\mathscr{O}\left(c_{n}^{M(M+1)-1}\right) \quad \text { and } \quad x_{n}^{2}=\mathscr{O}\left(c_{n}^{M^{p} / m^{p-2}+M^{p} / m^{p-1}-1}\right)
$$

for each $p \geq 2, p \in \mathbf{N}$. 
PROOF. From (3) we have

$$
c_{n-1} x_{n}^{2} \leq\left(x_{1}-x_{0}\right)^{2}+c_{1} x_{0}^{2}+c_{0} x_{1}^{2}+\sum_{i=1}^{n-1}\left(c_{i+1}-c_{i-1}\right) x_{i}^{2}, \quad n \in \mathbf{N}
$$

since $c_{i}>0$.

The last inequality can be writen in the form

$$
c_{n-1} x_{n}^{2} \leq C+\sum_{i=1}^{n-1} \frac{\left(c_{i+1}-c_{i-1}\right)}{c_{i+p-1}} c_{i+p-1} x_{i}^{2},
$$

where $C=\left(x_{1}-x_{0}\right)^{2}+c_{1} x_{0}^{2}+c_{0} x_{1}^{2}$ and $p \in \mathbf{N}$ is fixed.

Since $c_{n}>0,(5)$ is equivalent to

$$
c_{n+p-1} x_{n}^{2} \prod_{i=n}^{n+p-1} \frac{c_{i-1}}{c_{i}} \leq C+\sum_{i=1}^{n-1} \frac{\left(c_{i+1}-c_{i-1}\right)}{c_{i+p-1}} c_{i+p-1} x_{i}^{2},
$$

that is,

$$
\begin{aligned}
c_{n+p-1} x_{n}^{2} & \leq \prod_{i=n}^{n+p-1} \frac{c_{i}}{c_{i-1}}\left(C+\sum_{i=1}^{n-1} \frac{\left(c_{i+1}-c_{i-1}\right)}{c_{i+p-1}} c_{i+p-1} x_{i}^{2}\right) \\
& \leq M^{p}\left(C+\sum_{i=1}^{n-1} \frac{\left(c_{i+1}-c_{i-1}\right)}{c_{i+p-1}} c_{i+p-1} x_{i}^{2}\right) .
\end{aligned}
$$

By the condition of the theorem and the discrete Bellman-Gronwall lemma we obtain

$$
c_{n+p-1} x_{n}^{2} \leq C \exp \left(M^{p} \sum_{i=1}^{n-1} \frac{c_{i+1}-c_{i-1}}{c_{i+p-1}}\right)
$$

since $c_{i+1}-c_{i-1} \geq 0$.

Let us estimate the sum $\sum_{i=1}^{n-1}\left(c_{i+1}-c_{i-1}\right) / c_{i+p-1}$. First, we note that

$$
\sum_{i=1}^{n-1} \frac{c_{i+1}-c_{i-1}}{c_{i+p-1}}=\sum_{i=1}^{n-1} \frac{c_{i+1}-c_{i}}{c_{i+1}} \prod_{j=1}^{p-2} \frac{c_{i+j}}{c_{i+j+1}}+\sum_{i=1}^{n-1} \frac{c_{i}-c_{i-1}}{c_{i}} \prod_{j=1}^{p-1} \frac{c_{i+j-1}}{c_{i+j}} .
$$

By the condition of the theorem we have $c_{i} / c_{i+1} \leq 1 / m$ for all $i \in \mathrm{N}$. Thus, for $p \geq 2$, we have

$$
\sum_{i=1}^{n-1} \frac{c_{i+1}-c_{i-1}}{c_{i+p-1}} \leq \sum_{i=1}^{n-1} \frac{c_{i+1}-c_{i}}{c_{i+1}} \frac{1}{m^{p-2}}+\sum_{i=1}^{n-1} \frac{c_{i}-c_{i-1}}{c_{i}} \frac{1}{m^{p-1}}
$$


and for $p=1$,

$$
\sum_{i=1}^{n-1} \frac{c_{i+1}-c_{i-1}}{c_{i}} \leq \sum_{i=1}^{n-1} \frac{c_{i+1}-c_{i}}{c_{i+1}} M+\sum_{i=1}^{n-1} \frac{c_{i}-c_{i-1}}{c_{i}} .
$$

On the other hand, we have

$$
\sum_{i=1}^{n-1} \frac{c_{i+1}-c_{i}}{c_{i+1}} \leq \int_{c_{1}}^{c_{n}} \frac{d x}{x}
$$

and

$$
\sum_{i=1}^{n-1} \frac{c_{i}-c_{i-1}}{c_{i}} \leq \int_{c_{0}}^{c_{n-1}} \frac{d x}{x}
$$

By (6)-(8), we get

$$
\begin{aligned}
\sum_{i=1}^{n-1} \frac{c_{i+1}-c_{i-1}}{c_{i+p-1}} & \leq \frac{1}{m^{p-2}} \int_{c_{1}}^{c_{n}} \frac{d x}{x}+\frac{1}{m^{p-1}} \int_{c_{0}}^{c_{n-1}} \frac{d x}{x} \\
& =\frac{1}{m^{p-2}}\left(\ln c_{n}-\ln c_{1}\right)+\frac{1}{m^{p-1}}\left(\ln c_{n-1}-\ln c_{0}\right), \quad \text { for } p \geq 2
\end{aligned}
$$

and

$$
\sum_{i=1}^{n-1} \frac{c_{i+1}-c_{i-1}}{c_{i}} \leq M\left(\ln c_{n}-\ln c_{1}\right)+\left(\ln c_{n-1}-\ln c_{0}\right), \quad \text { for } p=1 .
$$

Hence, since $c_{n}$ is nondecreasing, we have

$$
\sum_{i=1}^{n-1} \frac{c_{i+1}-c_{i-1}}{c_{i+p-1}} \leq\left(\frac{1}{m^{p-2}}+\frac{1}{m^{p-1}}\right) \ln c_{n}+C, \text { for } p \geq 2
$$

and

$$
\sum_{i=1}^{n-1} \frac{c_{i+1}-c_{i-1}}{c_{i}} \leq(M+1) \ln c_{n}+C, \quad \text { for } p=1 .
$$

From all of the above we get

$$
c_{n+p-1} x_{n}^{2} \leq C \exp \left(M^{p}\left(\frac{1}{m^{p-2}}+\frac{1}{m^{p-1}}\right) \ln c_{n}+C\right), \quad \text { for } p \geq 2
$$

and $c_{n} x_{n}^{2} \leq C \exp \left(M(M+1) \ln c_{n}+C\right)$, for $p=1$. Thus we have

$$
c_{n+p-1} x_{n}^{2} \leq C c_{n}^{M^{p} / m^{p-2}+M^{p} / m^{p-1}} \leq C c_{n+p-1}^{M^{p} / m^{p-2}+M^{p} / m^{p-1}}, \text { for } p \geq 2 \text {, }
$$

that is,

$$
x_{n}^{2} \leq C C_{n+p-1}^{M^{p} / m^{p-2}+M^{p} / m^{p-1}-1}
$$

and $c_{n} x_{n}^{2} \leq C c_{n}^{M(M+1)}$, for $p=1$. From that the theorem follows readily. 
REMARK 1. Throughout the above proof we used $C$ to denote a positive constant, the value of which may vary from line to line.

COROLLARY 1. Let $c_{n}, n \in \mathbf{N}$, be a positive bounded nondecreasing sequence such that $1 \leq m \leq c_{n+1} / c_{n} \leq M<\infty, n \in \mathbf{N}$. Then all the solutions of (2) are bounded.

REMARK 2. Note that the condition $1 \leq m \leq c_{n+1} / c_{n} \leq M<\infty, n \in \mathrm{N}$, implies that the sequence $\left(c_{n}\right)$ in Theorem 1 and Corollary 1 is nondecreasing.

THEOREM 5. Let $d_{n}, n \in \mathbf{N}$, be a positive, unbounded, strictly concave sequence. Then Equation (1) where $b_{n}=-\left(d_{n+1}+d_{n-1}\right) / d_{n}$ has unbounded solution and $b_{n} \rightarrow$ -2 as $n \rightarrow \infty$.

PROOF. It is obvious that $d_{n}$ is an unbounded solution of (1). Since $d_{n}$ is a strictly concave sequence, that is, $d_{n+1}+d_{n-1}<2 d_{n}, n \in \mathbf{N}$, we have $d_{n+1}-d_{n}<d_{n}-d_{n-1}, n \in \mathbf{N}$. Thus the sequence $d_{n+1}-d_{n}$ is decreasing. Therefore there exists $\lim _{n \rightarrow \infty}\left(d_{n+1}-d_{n}\right)$ of finite or infinite value (that is, $-\infty)$. Let $\lim _{n \rightarrow \infty}\left(d_{n+1}-d_{n}\right)=d$. If $d<0$ from $d_{n}=d_{1}+\sum_{i=1}^{n-1}\left(d_{i+1}-d_{i}\right)$ we conclude that $d_{n}$ is negative for sufficiently large $n$. Then we arrive at a contradiction with the positivity assumption on $d_{n}$. So we have $d \geq 0$. Hence $d_{n+1} \geq d_{n}, n \in \mathbf{N}$. Since

$$
\lim _{n \rightarrow \infty}\left(d_{n+1}-d_{n}\right)=\lim _{n \rightarrow \infty} d_{n}\left(\frac{d_{n+1}}{d_{n}}-1\right)=d<+\infty
$$

and $\lim _{n \rightarrow \infty} d_{n}=+\infty$, we get $\lim _{n \rightarrow \infty}\left(d_{n+1} / d_{n}\right)=1$. It follows that

$$
\lim _{n \rightarrow \infty} b_{n}=-\lim _{n \rightarrow \infty} \frac{d_{n+1}+d_{n-1}}{d_{n}}=-2 \text {. }
$$

EXAMPLE 1. Consider the difference equation

$$
x_{n+1}-\frac{\ln (n+1)+\ln (n-1)}{\ln n} x_{n}+x_{n-1}=0, \quad n \geq 2 .
$$

This equation is of the form of (1) and obviously has solution $x_{n}=\ln n$ and $d_{n}=\ln n$, $n \geq 2$, satisfying the conditions of Theorem 5 .

EXAMPLE 2. Consider the difference equation

$$
x_{n+1}-\frac{(n+1)^{\alpha}+(n-1)^{\alpha}}{n^{\alpha}} x_{n}+x_{n-1}=0, \quad n \in \mathrm{N}, \alpha \in(0,1) .
$$

This equation is of the form of (1) and obviously it has solution $x_{n}=n^{\alpha}$. It is clear that $d_{n}=n^{\alpha}$ is a positive, unbounded and strictly concave sequence, since the function $f(x)=x^{\alpha}, \alpha \in(0,1)$, is such a function. 
By the "symmetry principle" we can obtain analogous theorems in the case $b_{n} \in$ $(0,2), n \in \mathbf{N}$.

\section{References}

[1] F. V. Atkinson, Discrete and Continuous Boundary Problems (Academic Press, 1964).

[2] R. Bellman, "The stability of solutions of linear differential equations", Duke Math. J. 10 (1943) 643-647.

[3] R. Bellman, Stability Theory of Differential Equations (McGraw-Hill, New York, 1953).

[4] F. S. De Blasi and J. Schinas, "On the stability of difference equations in Banach spaces", Analele stintificae ale Universitatii Al. Cuza, lasi Sectia la. Matematica 20 (1974) 65-80.

[5] T. Fort, Finite Differences and Difference Equations in the Real Domain (Oxford Univ. Press, London, 1948).

[6] T. Gronwall, "Note on the derivatives with respect to a parameter of the solutions of a system of differential equations", Ann. Math. 20 (2) (1919) 292-296.

[7] W. G. Kelly and A. C. Petersen, Difference Equations: An Introduction with Applications (Academic Press, Boston, 1991).

[8] E. Kurpinar and G. Sh. Guseinov, "The boundedness of solutions of the second-order difference equations", Indian J. Math. 37 (2) (1995) 113-122. 